\title{
Thin healthy women have a similar low bone mass to women with anorexia nervosa
}

\author{
D. Fernández-García ${ }^{1,2} *$, M. Rodríguez ${ }^{3}$, J. García Alemán ${ }^{1,2}$, J. M. García-Almeida ${ }^{1,2}$, \\ M. J. Picón ${ }^{1,2}$, F. Fernández-Aranda ${ }^{4}$ and F. J. Tinahones ${ }^{1,2}$ \\ ${ }^{1}$ Servicio de Endocrinología y Nutrición, Hospital Virgen de la Victoria, Málaga, Spain \\ ${ }^{2}$ CIBER Fisiopatología de la Obesidad y Nutrición (06/03), Instituto de Salud Carlos III, Málaga, Spain \\ ${ }^{3}$ Servicio de Reumatología, Hospital Carlos Haya, Málaga, Spain \\ ${ }^{4}$ Department of Psychiatry, University Hospital of Bellvitge, Barcelona, Spain
}

(Received 10 March 2008 - Revised 3 September 2008 - Accepted 14 January 2009 - First published online 23 March 2009)

An association between anorexia nerviosa (AN) and low bone mass has been demonstrated. Bone loss associated with AN involves hormonal and nutritional impairments, though their exact contribution is not clearly established. We compared bone mass in AN patients with women of similar weight with no criteria for AN, and a third group of healthy, normal-weight, age-matched women. The study included forty-eight patients with AN, twenty-two healthy eumenorrhoeic women with low weight (LW group; BMI $<18.5 \mathrm{~kg} / \mathrm{m}^{2}$ ) and twenty healthy women with BMI $>18.5 \mathrm{~kg} / \mathrm{m}^{2}$ (control group), all of similar age. We measured lean body mass, percentage fat mass, total bone mineral content (BMC) and bone mineral density in lumbar spine (BMD LS) and in total (tBMD). We measured anthropometric parameters, leptin and growth hormone. The control group had greater tBMD and BMD LS than the other groups, with no differences between the AN and LW groups. No differences were found in tBMD, BMD LS and total BMC between the restrictive $(n 25)$ and binge-purge type $(n 23)$ in AN patients. In AN, minimum weight $(P=0 \cdot 002)$ and percentage fat mass $(P=0.02)$ explained BMD LS variation $\left(r^{2} 0.48\right)$ and minimum weight $\left(r^{2} 0.42 ; P=0.002\right)$ for tBMD in stepwise regression analyses. In the LW group, BMI explained BMD LS $\left(r^{2} 0 \cdot 72 ; P=0 \cdot 01\right)$ and tBMD $\left(r^{2} 0.57 ; P=0 \cdot 04\right)$. We concluded that patients with AN had similar BMD to healthy thin women. Anthropometric parameters could contribute more significantly than oestrogen deficiency in the achievement of peak bone mass in AN patients.

Anorexia nervosa: Bone mineral density

Anorexia nervosa (AN) is a common disorder in adolescent girls, with a prevalence of $0.2-1.0 \%$ in Western societies ${ }^{(1)}$. The association of AN with low bone mineral density (BMD) has been conclusively and consistently demonstrated in adult women $^{(2-4)}$ as well as in adolescent girls ${ }^{(5-9)}$. Age at diagnosis and duration of AN determine the severity of bone mineral loss, whereas factors involved in the location of bone loss are not clear in $\mathrm{AN}^{(10,11)}$. Adolescence represents a critical period for the achievement of peak bone mass, as most bone mineral is accumulated during the teenage years, and deficits arising during this time may be permanent. Low peak bone mass is an important risk factor for osteoporosis and osteoporosis-related fracture after menopause. Therefore, an understanding of the factors that impact on bone density during this period is of particular importance.

Multiple hormone, endocrine and nutritional factors, such as weight, BMI and degree of undernutrition, affect bone metabolism in patients with $\mathrm{AN}^{(10,11)}$. To date, the main hormonal mechanisms known to lead to low bone mass in AN involve abnormalities in the growth hormone-insulin-like growth factor I axis. These hormones increase during puberty and stimulate the proliferation and differentiation of osteoblast precursors. Insulin-like growth factor I enhances bone formation and growth via effects on osteoblasts and collagen synthesis. Resistance to growth hormone with high growth hormone levels but low insulin-like growth factor I levels has been found in patients with $\mathrm{AN}^{(6,12-14)}$. High cortisol levels with a normal circadian cycle have been reported. Hypothalamic dysfunction or corticotropin-releasing hormone overproduction may contribute to hypercorticism ${ }^{(5,13)}$. Amenorrhoea is a diagnostic criterion for AN, and oestrogen deficiency has been described as a major source of bone loss in this condition. The mechanisms underlying this oestrogen deficiency involve multiple factors including hypothalamic dysfunction and weight loss ${ }^{(15,16)}$. Over recent years several other hormonal changes have been observed in AN patients, such as low levels of testosterone, leptin ${ }^{(7,14)}$, vitamin $\mathrm{D}^{(9)}$ and sex hormone-binding globulin ${ }^{(8)}$. The specific contribution of these factors to bone remodelling is not completely understood.

An association between AN and oestrogen deficiency has been reported, as has a correlation between bone loss and

Abbreviations: AN, anorexia nervosa; BMD, bone mineral density; BMD LS, bone mineral density in the lumbar spine; LW, low weight.

* Corresponding author: Dr Diego Fernandez García, fax + 34 951034016, email diegofernandezgarcia@hotmail.com 
the duration of amenorrhoea ${ }^{(6,17)}$. However, most studies failed to find total bone mass recovery with oestrogen-progestin therapy ${ }^{(18,19)}$. Oestrogen deficiency does not fully explain the loss of bone mass associated with $\mathrm{AN}$; malnutrition and nutrition-dependent factors therefore play a key role in the pathogenesis of bone loss associated with AN.

We hypothesised that at the beginning of the disease, when peak bone mass has not yet been reached, factors that involve bone mineral mass in AN patients are independent, at least in part, of oestrogen deficit and anthropometric variables and body composition will better predict bone mineral mass. To verify this hypothesis, we compared a group of patients with AN and oestrogen deficit with women of similar low weight with no criteria for AN (low-weight (LW) group; BMI $<18.5 \mathrm{~kg} / \mathrm{m}^{2}$ ) and therefore with no oestrogen deficit, and a third group of healthy, normal-weight, age-matched women (control group; BMI $18 \cdot 5-25 \mathrm{~kg} / \mathrm{m}^{2}$ ).

\section{Subjects and methods}

We studied forty-eight Caucasian adolescent girls who were referred by general practitioners to our unit for AN (aged 19 (SD 5) years). All of them met the Diagnostic and Statistical Manual of Mental Disorders 4th edition (DSM-IV) criteria for AN and secondary amenorrhoea (20 (SD 30) months) was present at the time of the study. None of them had been treated with $\mathrm{Ca}$ supplements, vitamin D preparations, hormone therapy, anti-resorptive therapy, thiazides, steroids, or other medications that might affect bone mass. The LW group was composed of twenty-two women, aged 18 (SD 4) years, recruited in primary care centres and who fulfilled the following criteria: BMI $<18.5 \mathrm{~kg} / \mathrm{m}^{2}$, eumenorrhoeic and no criteria for eating disorders. Women with co-morbid conditions known to be associated with secondary bone loss were excluded. After 5 years of follow-up, none presented any criteria for eating disorders. The control group included twenty healthy, Caucasian, female adolescents, aged 19.3 (SD 1.5) years, who were post-menarchal with regular menstrual periods and no history of eating disorders. Control subjects with a medical condition or receiving hormone or other medications known to affect bone metabolism were excluded. All the participants or their parents gave informed consent and the study was approved by the Research Committee of Virgen de la Victoria University Hospital (Malaga, Spain).

\section{Bone mineral density and body composition assessment}

BMD at the anterior-posterior lumbar spine L1-L4 (BMD LS) and total BMD and total bone mineral content were determined with dual-energy X-ray absorptiometry. The percentage of total body fat mass and lean body mass was quantified using the dual-energy X-ray absorptiometry method (Lunar DPX-L; Lunar Corp., Madison, WI, USA).

\section{Biochemical measurements}

Morning fasting samples of venous blood were taken. Serum was promptly separated and stored at $-80^{\circ} \mathrm{C}$ until assay. Serum growth hormone (Immunotech IRMA kit Beckman Coulter; manufacturer's reference level $<5 \mathrm{mU} / \mathrm{l})$ was assayed. Serum leptin (RIA; Nichols Institute Diagnostics,
Heston, Middlesex, UK; manufacturer's reference range for a normal BMI $\left(18 \cdot 5-25 \mathrm{~kg} / \mathrm{m}^{2}\right): 3 \cdot 7-11 \cdot 1 \mu \mathrm{g} / \mathrm{l}$; analytical sensitivity: $0.5 \mathrm{ng} / \mathrm{ml}$ ) was also measured.

\section{Statistical analysis}

For the descriptive analysis all values are presented as mean values and standard deviations. The comparison between quantitative variables was done with the ANOVA test. Intergroup comparisons were performed using the Student $t$ test and Mann-Whitney test in parametric and non-parametric comparisons, respectively. For the bivariate analysis we used simple linear (bivariate) correlations in all study groups to evaluate several interactions: anthropometric, nutritional and hormone parameters. Statistical significance was set at $P<0 \cdot 05$. A multivariate analysis was carried out to evaluate the impact of anthropometric and hormone parameters on BMD LS and total BMD in the AN patients and the LW group as well as a multivariate linear regression (stepwise addition). Inclusion of hormone, clinical and anthropometric variables in the analysis was based on the strength of correlation with BMD. All statistical analyses were performed with SPSS 13.0 (SPSS, Inc., Chicago, IL, USA).

\section{Results}

Table 1 shows the clinical characteristics of the study population. The control group had a higher total BMD and BMD LS than the AN and LW groups after adjusting for age, height and weight. No significant differences were found between the AN and LW groups in age, weight, height or BMI. Leptin levels and the percentage of fat mass were significantly lower (both $P<0.001$ ) in the AN group compared with the LW group, regardless of age. No significant differences were found in bone mass parameters between the two groups for total bone mineral content, total BMD or BMD LS. Regarding the two types of AN patients, the anthropometric parameters were similar in all the patients, regardless of whether they had the restrictive or the binge-purge variant of AN. No significant differences were found in total bone mineral content, total BMD or BMD LS, or in the other parameters, including leptin and growth hormone (Table 2).

Bivariate analysis in the AN group showed positive correlations between bone parameters and anthropometric parameters such as minimum weight, weight and BMI (Table 3). No correlations were found between bone markers, age and duration of AN and amenorrhoea. In the LW group, anthropometric parameters such as weight and BMI were correlated with total bone mineral content and total BMD. No correlation was found between BMD LS and anthropometric parameters (Table 3).

In the multivariate analysis (Table 4), an important influence of the anthropometric parameters in BMD was shown in the AN group. Minimum weight during the illness and percentage fat mass accounted for $48 \%$ of the BMD LS variance, while $42 \%$ of the total BMD variance was determined by minimum weight during the illness. In the LW group, up to $72 \%$ of the variance in BMD LS was due to BMI, and $57 \%$ of the total BMD variance was explained by the BMI. 
Table 1. Clinical characteristics of the study population

(Mean values and standard deviations)

\begin{tabular}{|c|c|c|c|c|c|c|}
\hline \multirow[b]{2}{*}{ Characteristic } & \multicolumn{2}{|c|}{ Control group } & \multicolumn{2}{|c|}{ AN group } & \multicolumn{2}{|c|}{ LW group } \\
\hline & Mean & SD & Mean & SD & Mean & SD \\
\hline Age (years) & $19 \cdot 30$ & 1.55 & $19 \cdot 00$ & $5 \cdot 1$ & 19.65 & $5 \cdot 34$ \\
\hline Height $(\mathrm{cm})$ & $160 \cdot 72$ & 5.83 & $159 \cdot 90$ & 6.69 & $159 \cdot 92$ & $5 \cdot 29$ \\
\hline Weight $(\mathrm{kg})$ & 54.4 & $5 \cdot 39$ & $44 \cdot 28^{*}$ & $5 \cdot 0$ & $43.44^{\star}$ & $5 \cdot 29$ \\
\hline $\operatorname{BMI}\left(\mathrm{kg} / \mathrm{m}^{2}\right)$ & $22 \cdot 30$ & 1.59 & $16 \cdot 13^{*}$ & 1.53 & $16 \cdot 65^{\star}$ & 1.02 \\
\hline Age at onset of illness (years) & - & & $16 \cdot 51$ & 3.31 & - & \\
\hline Duration of amenorrhoea (months) & - & & $20 \cdot 45$ & 31.4 & - & \\
\hline Total fat mass $(\%)$ & - & & $15 \cdot 37$ & $5 \cdot 63$ & $20 \cdot 63 \dagger$ & 3.40 \\
\hline Lean body mass (g) & - & & 35404.26 & $3706 \cdot 23$ & $34358 \cdot 10$ & $3915 \cdot 21$ \\
\hline Total bone mineral content $(\mathrm{g})$ & - & & $2010 \cdot 86$ & 383.73 & $1939 \cdot 60$ & $327 \cdot 10$ \\
\hline Bone mineral density in the lumbar spine $\left(\mathrm{g} / \mathrm{cm}^{2}\right)$ & 1.049 & 0.06 & $0.958^{*}$ & 0.13 & $0.956^{*}$ & 0.13 \\
\hline Total bone mineral density $\left(\mathrm{g} / \mathrm{cm}^{2}\right)$ & $1 \cdot 115$ & 0.08 & $1.075^{*}$ & 0.11 & $1.067^{*}$ & 0.09 \\
\hline Leptin (ng/ml) & 8.96 & 1.91 & $1.90^{*}$ & 1.76 & $4 \cdot 36^{\star} \dagger$ & $2 \cdot 01$ \\
\hline Growth hormone (ng/ml) & - & & 3.67 & 2.93 & 3.07 & 5 \\
\hline
\end{tabular}

AN, anorexia nervosa; LW, low weight.

${ }^{*}$ Mean value was significantly different from that of the control group $(P<0.001)$.

$\dagger$ Mean value was significantly different from that of the AN group $(P<0.001)$.

\section{Discussion}

Previous studies have shown that osteopenia occurs in $50 \%$ of young women with $\mathrm{AN}$ at both trabecular and cortical bone sites. Fractures are common in these young osteoporotic patients $^{(2-8)}$. These abnormalities result from the uncoupling of bone remodelling, including decreased bone formation and increased bone resorption ${ }^{(20)}$. Factors responsible for low bone mass in AN patients involve interrelated multiple hormone and nutritional impairments that damage osteoblast and/or osteoclast activities. This model differs from the postmenopausal osteoporosis model, where oestrogen deficiency only determines an increase in bone resorption. Several hormone mechanisms are currently known to lead to low bone mass. The specific contribution of these factors on bone remodelling, though, is not completely understood ${ }^{(5-9,13-16)}$.

In the present study, we compared three different groups of teenagers and young adult women, all of similar age: a group of healthy, normal-weight women, a group of eumenorrhoeic women with BMI $<18.5 \mathrm{~kg} / \mathrm{m}^{2}$ with no criteria for AN (LW group) and a group of AN patients having a mean of 18 months' disease evolution and of similar weight to the LW group. The study of these three different groups enabled us to evaluate the independent contribution of oestrogen deficit and weight to bone mineral mass at the onset of the disease. The association between the hypo-oestrogenic state and early onset bone loss in $\mathrm{AN}$ patients ${ }^{(21)}$ and the need to reach normal oestrogen levels during this period of bone mass acquisition $^{(22)}$ have been reported previously. Klibanski et al. showed in a prospective study that oestrogen administration was effective in preventing progressive osteopenia in lean AN patients only when associated with weight gain ${ }^{(16)}$. The reason why oestrogen replacement alone is not sufficient to promote bone accretion in young patients with $\mathrm{AN}$ is currently unknown, but it may well mean that proper nutrition and recovery of normal circulating bone trophic factors such as insulin-like growth factor I are required for oestrogen action on bone.

Table 2. Differences between restrictive and purgative types within the anorexia nervosa group (Mean values and standard deviations)

\begin{tabular}{|c|c|c|c|c|c|}
\hline & \multicolumn{2}{|c|}{ Purgative ( $n$ 23) } & \multicolumn{2}{|c|}{ Restrictive ( $n$ 25) } & \multirow[b]{2}{*}{ Significance } \\
\hline & Mean & SD & Mean & SD & \\
\hline Minimum weight $(\mathrm{kg})$ & $39 \cdot 18$ & 3.72 & 38.5 & 5.59 & NS \\
\hline Age at onset of illness (years) & $16 \cdot 85$ & 2.47 & $16 \cdot 32$ & 3.8 & NS \\
\hline Duration of amenorrhoea (months) & 29.4 & 44.91 & $16 \cdot 00$ & $19 \cdot 16$ & NS \\
\hline Weight (kg) & 41.52 & 4.98 & $41 \cdot 13$ & $5 \cdot 28$ & NS \\
\hline Height $(m)$ & 1.60 & 0.06 & 1.59 & 0.069 & NS \\
\hline $\mathrm{BMI}\left(\mathrm{kg} / \mathrm{m}^{2}\right)$ & $16 \cdot 15$ & 1.59 & $16 \cdot 12$ & 1.53 & NS \\
\hline Leptin (ng/ml) & $2 \cdot 8$ & $2 \cdot 32$ & 1.33 & 1.01 & NS \\
\hline Growth hormone (ng/ml) & 4.91 & 3.67 & 3.3 & $2 \cdot 64$ & NS \\
\hline Bone mineral density in the lumbar spine $\left(\mathrm{g} / \mathrm{cm}^{2}\right)$ & 0.948 & 0.111 & 0.964 & 0.121 & NS \\
\hline Total bone mineral density $\left(\mathrm{g} / \mathrm{cm}^{2}\right)$ & 1.073 & 0.099 & 1.076 & 0.105 & NS \\
\hline Total bone mineral content $(\mathrm{g})$ & $2012 \cdot 27$ & $258 \cdot 79$ & $2009 \cdot 45$ & $353 \cdot 17$ & NS \\
\hline Lean body mass $(\mathrm{g})$ & 34877.44 & 2778.73 & 35787.41 & 4280.33 & NS \\
\hline Total fat mass $(\%)$ & $16 \cdot 60$ & $6 \cdot 15$ & 14.47 & $5 \cdot 18$ & NS \\
\hline
\end{tabular}


Table 3. Correlation analysis of the biological variables studied (Pearson's $r$ ) in the anorexia nervosa (AN) and low-weight (LW) groups

\begin{tabular}{|c|c|c|c|c|c|c|}
\hline & \multicolumn{2}{|c|}{ BMD LS } & \multicolumn{2}{|c|}{ Total BMD } & \multicolumn{2}{|c|}{ Total BMC } \\
\hline & AN & LW & AN & LW & AN & LW \\
\hline Age & 0.01 & 0.27 & 0.06 & 0.28 & 0.13 & 0.41 \\
\hline Illness duration & -0.08 & - & -0.14 & - & -0.12 & - \\
\hline Minimum weight & $0.60^{\star *}$ & - & $0.69^{\star *}$ & - & $0.83^{\star *}$ & - \\
\hline Weight & $0.46^{\star}$ & 0.47 & $0.59^{\star *}$ & $0.32^{*}$ & $0.76^{\star *}$ & $0.84^{\star \star}$ \\
\hline BMI & $0.51^{\star *}$ & 0.53 & $0.49^{\star *}$ & $0.54^{*}$ & $0.45^{\star *}$ & $0.73^{\star \star}$ \\
\hline Months of amenorrhoea & -0.06 & - & -0.11 & - & -0.21 & - \\
\hline Percentage total fat mass & 0.24 & 0.35 & 0.14 & 0.22 & 0.16 & 0.27 \\
\hline Lean body mass & 0.36 & 0.32 & $0.54^{\star \star}$ & 0.22 & $0.76^{\star *}$ & $0.84^{\star \star}$ \\
\hline Leptin & 0.02 & -0.01 & 0.01 & 0.02 & 0.06 & -0.23 \\
\hline Growth hormone & $0 \cdot 13$ & $0 \cdot 26^{*}$ & $0 \cdot 19$ & $0.29^{*}$ & $0 \cdot 16$ & $0.69^{* \star}$ \\
\hline
\end{tabular}

BMD LS, bone mineral density in the lumbar spine; BMD, bone mineral density; BMC, bone mineral content. ${ }^{\star} P<0.05,{ }^{\star \star} P<0.001$.

The first outstanding result in the present study was that the $\mathrm{LW}$ women had the same bone mineral mass as the AN patients. Both groups had lower total BMD and BMD LS than healthy women of a similar age. In the present study, BMD LS and total BMD in AN and LW patients were significantly related to anthropometric variables, though the duration of amenorrhoea did not explain the changes in BMD in the AN patients. Consequently, these results suggest that anthropometric parameters could contribute more significantly than oestrogen deficit in the AN patients of short evolution. A similar decrease in BMD in the LW group and the AN patients strengthens this theory. The relationship between BMI and BMD in AN patients and healthy women has been reported previously. Several studies have demonstrated that body weight in healthy premenopausal women is an independent and determinant factor for adequate peak bone mass in this population $^{(23-25)}$. Bone loss is inversely correlated with weight in AN patients. Greater weight $\operatorname{loss}^{(26,27)}$ and a longer duration of the low weight state ${ }^{(28)}$ produce a higher bone loss. Similarly, there was a correlation with lean mass, which is supported by previous studies where the loss of

Table 4. Multiple regression analysis in the anorexia nervosa (AN) and low-weight (LW) groups*

\begin{tabular}{|c|c|c|c|c|c|c|}
\hline \multirow[b]{2}{*}{ Variables } & \multicolumn{3}{|c|}{ AN } & \multicolumn{3}{|c|}{ LW } \\
\hline & $R^{2}$ & $\begin{array}{c}\text { SE } \\
(\beta)\end{array}$ & $P$ & $R^{2}$ & $\begin{array}{r}\text { SE } \\
(\beta)\end{array}$ & $P$ \\
\hline \multicolumn{7}{|l|}{ BMD LS } \\
\hline \multicolumn{7}{|c|}{ Independent variables } \\
\hline Minimum weight & 0.48 & 0.46 & 0.002 & & & \\
\hline$\%$ Fat mass & & 0.44 & 0.02 & & & \\
\hline BMI & & & & 0.72 & 0.85 & 0.01 \\
\hline \multicolumn{7}{|l|}{ Total BMD } \\
\hline \multicolumn{7}{|l|}{ Independent variables } \\
\hline $\begin{array}{l}\text { Minimum weight } \\
\text { BMI }\end{array}$ & 0.42 & 0.65 & 0.002 & 0.57 & 0.72 & 0.04 \\
\hline
\end{tabular}

lean mass was considered an important prognostic factor to predict the bone mass loss, with a significant correlation between lean mass and BMD LS and total $\mathrm{BMD}^{(29,30)}$.

The present study revealed no differences between the two forms of AN, the restrictive and the binge-purge types, either in bone or anthropometric parameters. Previous studies comparing the bone density between the two different forms of AN have yielded inconsistent findings ${ }^{(31-34)}$. The present results are consistent with data suggesting that the degree of underweight is an important predictor of low BMD, whereas other behaviour such as bingeing and purging seems to be of relatively minor importance. Discrimination between the two AN types is based on behavioural symptoms (for example, purging) that frequently change during the course of the illness ${ }^{(35,36)}$. This heterogeneity is associated with a masking of the differences in effects that restrictive and binge-purge eating behaviours might have on bone parameters.

Leptin exerts a critical role in the regulation of body energy balance and as a protective factor inhibiting bone resorption $^{(37-39)}$. However, the exact mechanism underlying the effect of variation in this adipokine on bone anomalies in patients with AN is still not clear. The decrease in leptin levels observed in AN patients could contribute to BMD loss due to the reduction in bone formation ${ }^{(10)}$. Additionally, the correlation between leptin and bone resorption markers in AN patients during the refeeding process suggests a possible cause-effect relationship between serum levels of leptin and bone metabolism ${ }^{(40)}$. Conversely, leptin deficiency in AN was not an independent predictor of BMD after controlling for other nutrition-dependent factors such as BMI. Despite the marked decrease in serum leptin levels in the AN group compared with the LW group no changes in BMD were revealed.

The present study has several limitations. First, hypooestrogenism was defined by a surrogate value, duration of amenorrhoea, because oestradiol or gonadotrophin serum levels were unavailable. Second, in our study, comparison groups were small. However, there are not many papers reporting differences between patients with AN, healthy women with LW and women with normal weight. Third, BMD could not be adjusted for 25-hydroxyvitamin D levels, an important cause in the pathophysiology of bone alterations in AN. 
In conclusion, the present results support the hypothesis that anthropometric parameters could contribute more significantly to low bone mass than oestrogen deficiency in AN patients of short evolution. Therefore, teenagers and adult women with low weight have an increased risk for low bone mass similar to that of AN patients.

\section{Acknowledgements}

The authors wish to thank all the subjects and Dr Fernando Cardona for their collaboration. D. F.-G. and F. J. T. were investigators, managed the data and wrote the manuscript; J. G.-A., J. M. G.-A., M. R. and M. J. P. were the clinical investigators and helped in the making of the database; F. F.-A. was an investigator who provided advice.

There are no conflicts of interest to declare.

\section{References}

1. Von Ranson K, Iacono W \& McGue M (2002) Disordered eating and substance abuse in an epidemiological sample. I. Associations within individuals. Int $J$ Eat Disord 31, 389-403.

2. Rigotti N, Neer R, Skates S, et al. (1991) The clinical course of osteoporosis in anorexia nervosa. A longitudinal study of cortical bone mass. JAMA 265, 1133-1138.

3. Bolton J, Patel S, Lacey J, et al. (2005) A prospective study of changes in bone turnover and bone density associated with regaining weight in women with anorexia nervosa. Osteoporos Int 16, 1955-1962.

4. Grinspoon S, Miller K, Coyle C, et al. (1999) Severity of osteopenia in estrogen-deficient women with anorexia nervosa and hypothalamic amenorrhea. J Clin Endocrinol Metab 84, 2049-2055.

5. Misra M, Aggarwal A, Miller KK, et al. (2004) Effects of anorexia nervosa on clinical, hematologic, biochemical, and bone density parameters in community-dwelling adolescent girls. Pediatrics 14, 1574-1583.

6. Soyka L, Grinspoon S, Levitsky L, et al. (1999) The effects of anorexia nervosa on bone metabolism in female adolescents. $J$ Clin Endocrinol Metab 84, 4489-4496.

7. Soyka L, Misra M, Frenchman A, et al. (2002) Abnormal bone mineral accrual in adolescent girls with anorexia nervosa. J Clin Endocrinol Metab 87, 4177-4185.

8. Schneider M, Fisher M, Weinerman S, et al. (2002) Correlates of low bone density in females with anorexia nervosa. Int J Adolesc Med Health 14, 297-306.

9. Turner J, Bulsara M, McDermott B, et al. (2001) Predictors of low bone density in young adolescent females with anorexia nervosa and other dieting disorders. Int $J$ Eat Disord 30, $245-251$.

10. Munoz MT \& Argente $\mathbf{J}$ (2002) Anorexia nervosa in female adolescents: endocrine and bone mineral density disturbances. Eur J Endocrinol 147, 275-286.

11. Legroux-Gerot I, Vignau J, Collier F, et al. (2005) Bone loss associated with anorexia nervosa. Joint Bone Spine 72, 489-495.

12. Grinspoon S, Baum H, Lee K, et al. (1996) Effects of short-term recombinant human insulin-like growth factor I administration on bone turnover in osteopenic women with anorexia nervosa. $J$ Clin Endocrinol Metab 81, 3864-3870.

13. Misra M, Miller K, Cord J, et al. (2007) Relationships between serum adipokines, insulin levels and bone density in girls with anorexia nervosa. J Clin Endocrinol Metab 92, 2046-2052.
14. Misra M, Miller KK, Bjornson J, et al. (2003) Alterations in growth hormone secretory dynamics in adolescent girls with anorexia nervosa and effects on bone metabolism. J Clin Endocrinol Metab 88, 5615-5623.

15. Herzog W, Minne H, Deter C, et al. (1993) Outcome of bone mineral density in anorexia nervosa patients 11.7 years after first admission. J Bone Miner Res 8, 597-605.

16. Klibanski A, Biller BM, Schoenfeld DA, et al. (1995) The effects of estrogen administration on trabecular bone loss in young women with anorexia nervosa. J Clin Endocrinol Metab 80, 898-904.

17. Grinspoon S, Thomas E, Pitts S, et al. (2000) Prevalence and predictive factors for regional osteoporosis in women with anorexia nervosa. Ann Intern Med 133, 790-794.

18. Bolton JGF \& Patel S (2001) Osteoporosis in anorexia nervosa. J Psychosom Res 50, 177-178.

19. Karlsson MK, Weigall SJ, Duan Y, et al. (2000) Bone size and volumetric density in women with anorexia nervosa receiving estrogen replacement therapy and in women recovered from anorexia nervosa. J Clin Endocrinol Metab 85, 3177-3182.

20. Caillot-Augusseau A, Lafage-Proust MH, Margaillan P, et al. (2000) Weight gain reverses bone turnover and restores circadian variation of bone resorption in anorexic patients. Clin Endocrinol 52, 113-121.

21. Heer M, Mika C, Grzella I, et al. (2004) Bone turnover during inpatient nutritional therapy and outpatient follow-up in patients with anorexia nervosa compared with that in healthy control subjects. Am J Clin Nutr 80, 774-781.

22. Hergenroeder AC (1995) Bone mineralization, hypothalamic amenorrhea, and sex steroid therapy in female adolescents and young adults. $J$ Pediatr 126, 683-689.

23. Rubin LA, Hawker GA, Peltekova VD, et al. (1999) Determinants of peak bone mass: clinical and genetic analyses in a young female Canadian cohort. $J$ Bone Miner Res 14, 633-643.

24. Valdimarsson O, Kristinsson JO, Stefansson SO, et al. (1999) Lean mass and physical activity as predictors of bone mineral density in 16-20-year old women. J Intern Med 245, 489-496.

25. Hawker GA, Jamal SA, Ridout R, et al. (2002) A clinical prediction rule to identify premenopausal women with low bone mass. Osteoporos Int 13, 400-406.

26. Baker D, Roberts R \& Towell T (2000) Factors predictive of bone mineral density in eating-disordered women: a longitudinal study. Int J Eat Disord 27, 29-35.

27. Wong S, Au B, Lau E, et al. (2004) Osteoporosis in Chinese patients with anorexia nervosa. Int J Eat Disord 36, 104-108.

28. Hotta M, Shibasaki T, Sato K, et al. (1998) The importance of body weight history in the occurrence and recovery of osteoporosis in patients with anorexia nervosa: evaluation by dual X-ray absorptiometry and bone metabolic markers. Eur J Endocrinol 139, 276-283.

29. Lucas AR, Melton LJ III, Crowson CS, et al. (1999) Long-term fracture risk among women with anorexia nervosa: a population-based cohort study. Mayo Clin Proc 74, 972-977.

30. Wong JC, Lewindon P, Mortimer R, et al. (2001) Bone mineral density in adolescent females with recently diagnosed anorexia nervosa. Int $J$ Eat Disord 29, 11-16.

31. Zipfel S, Seibel MJ, Lowe B, et al. (2001) Osteoporosis in eating disorders: a follow-up study of patients with anorexia and bulimia nervosa. J Clin Endocrinol Metab 86, 5227-5233.

32. Andersen AE, Woodward PJ \& LaFrance N (1995) Bone mineral density of eating disorder subgroups. Int J Eat Disord 18, 335-342.

33. Andersen AE, Watson T \& Schlechte J (2000) Osteoporosis and osteopenia in men with eating disorders. Lancet $\mathbf{3 5 5}$, 1967-1968. 
34. Goebel G, Schweiger U, Kruger R, et al. (1999) Predictors of bone mineral density in patients with eating disorders. Int $J$ Eat Disord 25, 143-150.

35. Nielsen S \& Palmer B (2003) Diagnosing eating disorders AN, BN and the others. Acta Psychiatr Scand 108, 161-162.

36. Fairburn CG \& Harrison PJ (2003) Eating disorders. Lancet 361, 407-416.

37. Muñoz MT, Morande G, García-Centenera JA, et al. (2002) The effects of estrogen administration on bone mineral density in adolescents with anorexia nervosa. Eur $J$ Endocrinol 146, $45-50$.
38. Grinspoon S, Gulick T, Askari H, et al. (1996) Serum leptin levels in women with anorexia nervosa. J Clin Endocrinol Metab 81, 3861-3863.

39. Herpertz S, Albers N, Wagner R, et al. (2000) Longitudinal changes of circadian leptin, insulin and cortisol plasma levels and their correlation during refeeding in patients with anorexia nervosa. Eur J Endocrinol 142, 373-379.

40. Audi L, Vargas DM, Gussinye M, et al. (2002) Clinical and biochemical determinants of bone metabolism and bone mass in adolescent female patients with anorexia nervosa. Pediatr Res 51, 497-504. 